\title{
Dance Clubbing on MDMA and during Abstinence from Ecstasy/MDMA: Prospective Neuroendocrine and Psychobiological Changes
}

\author{
A.C. Parrott ${ }^{a} \quad$ J. Lock ${ }^{a} \quad$ A.C. Conner ${ }^{c} \quad$ C. Kissling ${ }^{b} \quad$ J. Thome ${ }^{b}$

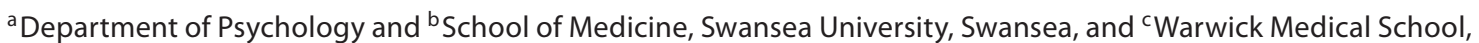 \\ University of Warwick, Warwick, UK
}

\section{Key Words}

3,4-Methylenedioxymethamphetamine (MDMA) • Ecstasy • Cortisol • Testosterone $\cdot$ Neuroendocrine $\cdot$ Temperature Energy $\cdot$ Stress $\cdot$ Serotonin

\section{Abstract}

Background/Aims: The present study is the first to prospectively compare a group of recreational Ecstasy users when dance clubbing on 3,4-methylenedioxymethamphetamine (MDMA) and when clubbing during abstinence from Ecstasy/MDMA. Methods: Twelve normal healthy volunteers (mean age $=23.2$ years) were assessed at a Saturday night dance club under self-administered MDMA. On the other weekend they went to the same dance club without taking MDMA (order counterbalanced). Both conditions involved 5 test sessions conducted at similar times: pre-drug baseline, $1 \mathrm{~h}$ post-drug clubbing, $2.5 \mathrm{~h}$ post-drug clubbing, and 2 and 4 days later. The assessments included body and ambient temperature, physical activity (pedometer), as well as selfratings for mood state, physical activity, thermal comfort and thirst. Saliva samples were analyzed for MDMA, cortisol and testosterone. Results: The cortisol levels increased significantly by $800 \%$ when dance clubbing on MDMA, while testosterone increased significantly by $75 \%$; neither neuroendocrine measure was altered during abstinence. Saliva analyses confirmed the presence of MDMA when dancing
\end{abstract}

\section{KARGER}

Fax +41613061234

E-Mail karger@karger.ch

www.karger.com

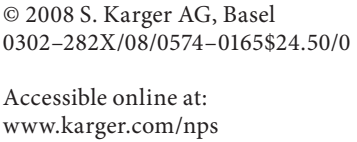

on Ecstasy and its absence when dancing off Ecstasy. The pedometer values and self-rated levels of dancing were similar at both weekends. Hot and cold flushes and feeling hot increased significantly under MDMA. The mean body temperature did not change significantly, although there was a borderline trend for increased values after MDMA. Feelings of happiness and excitement increased under MDMA, although they were not significantly greater than when clubbing during abstinence. Conclusions: Neurohormonal release may be an important part of the acute MDMA experience. The large cortisol increase provides further data on the bioenergetic stress model of recreational Ecstasy/ MDMA.

Copyright $\odot 2008$ S. Karger AG, Basel

\section{Introduction}

MDMA (3,4-methylenedioxymethamphetamine) or 'Ecstasy' is used as an illicit drug in many countries worldwide [1-6]. MDMA is a powerful indirect agonist for serotonin (5-hydroxytryptamine), and it also stimulates dopamine, noradrenaline and other neurotransmitter activity [7-9]. Further downstream effects of signalling cascades and gene expression processes may also be important [10]. The acute monoaminergic boost is thought to underlie the positive mood effects of MDMA,
Professor A.C. Parrott

Department of Psychology

Swansea University

Swansea SA2 8PP (UK)

Tel. +44 1792295 271, Fax +44 1792295 679, E-Mail a.c.parrott@swansea.ac.uk 
with feelings of euphoria, elation, vigour and well-being $[7,11,12]$; hence its street names as 'Ecstasy' or the 'love drug' [5, 13-15]. The days following recreational Ecstasy/ MDMA are accompanied by a rebound period of neurotransmitter depletion, when low moods such as lethargy and depression predominate [16-18]. The wider psychobiological changes during this recovery period can include reduced appetite, impaired sleep and aggressiveness [5, 11, 17, 19-22].

Neuroendocrine functioning is also affected by acute and chronic MDMA, through the acute release of serotonin and noradrenalin and the resulting stimulation of hypothalamus-pituitary-adrenal (HPA) axis activity. Dumont and Verkes [2] noted that cortisol was the most widely studied of the neurohormones. Furthermore 11 of the 12 laboratory studies which had assessed cortisol found that MDMA led to a significant increase. Harris et al. [23] noted that $0.5 \mathrm{mg} / \mathrm{kg}$ of MDMA led to an increase over baseline of around $100 \%$, whereas $1.5 \mathrm{mg} / \mathrm{kg}$ of MDMA caused a $150 \%$ percentage increase (i.e. from 11 to $28 \mu \mathrm{g} / \mathrm{dl}$ at $2 \mathrm{~h}$, with values partially derived from visual inspection of figure 2 in Harris et al. [23]). Several other studies have reported similar acute increases in cortisol [24-28]. In chronic terms, Gerra et al. [29] investigated neuroendocrine functioning in drug-free recreational Ecstasy/MDMA users. They were found to have significantly higher basal cortisol than non-users, together with a blunted cortisol response to stress. This allowed the authors to conclude that regular MDMA usage had led to a 'complex neuroendocrine dysfunction'. Cortisol changes were also evident in an investigation of tobacco smoking and abstinence in recreational Ecstasy/MDMA users and controls [30]. The Ecstasy users demonstrated a stronger neuroendocrine response to nicotine deprivation, which was consistent with the conclusion of Gerra et al. [29] that regular MDMA can disrupt neuroendocrine integrity. The theoretical importance of cortisol is that it is an energetic stress hormone and may therefore underlie some of MDMA's actions as an acute metabolic stressor [11]. Hence it was deemed important to generate further data on the cortisol levels in Ecstasy/MDMA users, especially in the physically demanding environment of dance clubs. Cortisol and testosterone have close physiological links via the HPA and hypothalamus-pituitary-gonadal axes [50]. Yet testosterone does not seem to have been investigated in previous human or animal MDMA research, which is surprising given its common usage for sexual enhancement $[14,52]$. Any changes in testosterone may also be important for understanding its behavioural effects on sex and aggressiveness [14, 17, 52].
The recreational use of MDMA is strongly associated with dance clubbing $[1,4,14,31-35]$. Clubs and raves tend to be noisy and crowded, with dancing often continuing over prolonged periods. Dance clubbing therefore places high levels of energetic demand on the human organism, and this may be exacerbated by the co-use of stimulant drugs. At a large Dutch rave, Suy et al. [36] reported that physical exhaustion was the most common reason for referral to the paramedic support team. Neuroendocrine alteration may be important here, since any changes in cortisol and testosterone caused by MDMA (see previous paragraph) may be further increased by the additional physical stress of dance clubbing. In the laboratory, MDMA leads to general sympathetic activation, with an increased metabolic rate, raised body temperature and subjective reports of hyperthermia [37]. The temperature changes may be part of a wider pattern of acute metabolic activation, which has elements of the serotonin syndrome [31]. Feeling hot is therefore a typical experience for many Ecstasy/MDMA-using dance clubbers. However it is not universally reported, and there may be important variation in these body temperature changes and in their psychobiological consequences $[1,4]$. Some studies have found a significant increase in core body temperature following acute MDMA [37,38], whereas others have observed an unchanged body temperature $[26,39,40]$. There is also variation in the findings from dance clubbers. Irvine et al. [41] noted a non-significant group trend for higher temperature, along with a borderline trend for a positive correlation between temperature increases and plasma MDMA levels, in Ecstasy users tested several hours after partying. Cole et al. [42] found no body temperature effects in recreational stimulant (including Ecstasy) users at a dance club. Parrott and Young [43] found a significantly higher body temperature in current Ecstasy/MDMA users while dance clubbing, compared to the non-user controls, with former Ecstasy users showing intermediate values. So while a core aim of the current study was to investigate the acute neuroendocrine changes in this physically stressful situation, another objective was to assess the thermal aspects of MDMA use in dance clubbers. Finally, the present study utilized the novel design of Ecstasy/MDMA users acting as their own controls. Each volunteer agreed to be monitored whilst dance clubbing at the same club venue over 2 weekends and to abstain from taking Ecstasy/MDMA on one of these occasions. The statistically powerful 'within-group' design is standard in many areas of psychopharmacology research, although it has not been previously employed with recreational Ecstasy/MDMA users. The aim was to 
generate further empirical data on the neuropsychobiological effects of this CNS stimulant in the dance club environment.

\section{Methods}

\section{Participants}

The nature of this study meant that it was important to have thoroughly screened and well-motivated participants. None of the volunteers were paid, nor was there any advertising. Instead all of the participants were friends or acquaintances of the key research worker (J.L.). The 12 participants comprised 8 males and 4 females. Most were university students, and the others were in paid employment. The non-inclusion criteria were diagnosis of a psychiatric disorder or a medical condition requiring medication. All were regular Ecstasy/MDMA-using dance clubbers from 2 British towns, Swansea and Brighton. The age range was 18-48 years, with an overall mean of 23.2, since all but one of the participants were aged 18-25 years. The Swansea group consisted of 4 males and 1 female, while the Brighton group comprised 4 males and 3 females. Each group was tested together with everyone being assessed under the same on-drug or off-drug condition; the order was counterbalanced between the groups.

\section{Drug Conditions}

All the participants reported that they normally used Ecstasy/ MDMA while dance clubbing at weekends. For this study they agreed to be tested while clubbing over 2 successive weekends. On 1 weekend they would behave as they would do normally, while on the other they would refrain from self-administering any illicit stimulant drug such as Ecstasy/MDMA. Saliva samples were taken at the club and later tested for drug presence (see later). This report covers the 12 participants who gave positive MDMA results for the normal weekend and negative MDMA results for the abstinence weekend. The participants also agreed to limit their use of all other psychoactive drugs, both legal and illicit, during the 2 weekends and consented not to take any other stimulant drugs during the 2 -week period of testing. They were asked to refrain from using ecstasy for a week prior to the study and to abstain from all other drug use for at least $24 \mathrm{~h}$ before testing. Each participant also completed the University of East London drug use questionnaire for lifetime usage of recreational drugs [44], followed by a more detailed set of questions about Ecstasy/MDMA usage patterns [45].

\section{Temperature and Physical Activity}

Body temperature was measured using a commercial TH-809 infrared ear thermometer. Each participant was seated and instructed not to move until a constant temperature had been established. Ambient temperature was recorded using a mercury thermometer nearby. Physical activity was assessed by means of a pedometer: the Yamax SW-200 Digi-Walker. Each participant set the pedometer to 0 after entering the club, and the cumulative activity reading was recorded at the end of the second test session.

\section{Subjective Self-Ratings}

These were assessed using a 23-item self-rating questionnaire, covering 4 broad areas, with the questions derived from previous studies $[18,43,46]$. The general activity questions included the extent of dancing, frequency of resting, and time spent drinking water and other fluids. The mood state questions covered normal feelings of excitement, happiness, relaxation, anxiety, depression, boredom, unpleasantness, clear-headedness, quick-wittedness, sociability and social withdrawal (note: the scales referred to normal moods and did not indicate the clinical/psychiatric status). The bodily symptom questions covered physical tiredness, impaired concentration, feeling energetic and memory problems. The temperature and thirst questions included feeling hot, feeling cold, hot and cold flushes, sweating and thirst. The responses were evaluated on a forced-choice scale with 5 alternatives: not at all, rarely, sometimes, frequently, all the time.

\section{Neuroendocrine and Drug Measures}

These were assessed by saliva sampling. The samples were collected using proprietary SaliCaps (IBL-Hamburg) made of ultrapure polypropylene which minimizes the absorption of analytes such as cortisol or testosterone. The samples were cooled in a refrigerator at $4^{\circ} \mathrm{C}$ within $4 \mathrm{~h}$ of collection and subsequently frozen for storage. The ortisol and testosterone levels were assessed using the commercial IBL-Hamburg test systems. The MDMA levels in saliva were quantified with a commercial kit based on ELISA technology (Cozart, UK). This competitive enzyme immunoassay is specific for MDMA/methamphetamine. Although it does not differentiate between methamphetamine and MDMA, none of our Ecstasy users reported that they had ever knowingly taken methamphetamine.

\section{Procedure}

The participants were assessed in 2 groups, one in the order MDMA-abstinence and the other in the order abstinence-MDMA. This counterbalancing controlled for learning and fatigue effects. The normal clubbing (on-MDMA) and clubbing when abstinent (off-MDMA) sessions were held 1 week apart. Each week there were 5 test sessions: pre-drug, $1 \mathrm{~h}$ after drug while clubbing, $2.5 \mathrm{~h}$ after drug while clubbing, 2 days later and 4 days later. The pre-drug baseline was at the experimenter's house in the early evening, around 30-60 min before going to the dance club venue. The post-drug sessions at the dance club were conducted at a prearranged venue in the club, between the hours of 11 p.m. and 2 a.m. The last 2 sessions took place either at the experimenter's or volunteer's home. At the dance club, each participant was allocated a specific time to be tested, with the data collection being undertaken by 2 experimenters. Each participant was assessed at approximately the same time both weekends, in order to control for potential circadian influences. The test sessions normally took around $5 \mathrm{~min}$ to complete, although this could take up to $15 \mathrm{~min}$ when saliva production was difficult. The order of testing was held constant at every session: ear temperature, ambient temperature, self-rating questionnaire and saliva sampling.

\section{Ethical Permission}

The study protocol followed the principles of the Helsinki Agreement and was passed by the ethics committee. Each participant was required to sign a written agreement before the study commenced. This noted that each volunteer was free to withdraw at any time without giving any reason and that they had the option of not answering a question if they so wished (1 participant withdrew after completing 3 sessions, and her incomplete data 
Table 1. Neuroendocrine and thermal assessments for 12 recreational Ecstasy/MDMA users before, during and after dance clubbing

\begin{tabular}{|c|c|c|c|c|c|c|c|c|c|c|c|}
\hline \multirow[t]{2}{*}{$\begin{array}{l}\text { Assessed } \\
\text { measure }\end{array}$} & \multirow[t]{2}{*}{$\begin{array}{l}\text { MDMA } \\
\text { status }\end{array}$} & \multirow[t]{2}{*}{$\begin{array}{l}\text { Pre-drug } \\
\text { (1) }\end{array}$} & \multirow{2}{*}{$\begin{array}{l}1 \mathrm{~h} \\
\text { after drug } \\
(2)\end{array}$} & \multirow{2}{*}{$\begin{array}{l}2.5 \mathrm{~h} \\
\text { after drug } \\
\text { (3) }\end{array}$} & \multirow{2}{*}{$\begin{array}{l}2 \text { days } \\
\text { after drug } \\
(4)\end{array}$} & \multirow{2}{*}{$\begin{array}{l}4 \text { days } \\
\text { after drug } \\
\text { (5) }\end{array}$} & \multirow{2}{*}{$\begin{array}{l}\mathrm{p} \text { value } \\
\text { ANOVA } \\
\text { (session } \\
\text { effects) }\end{array}$} & \multicolumn{4}{|c|}{$\begin{array}{l}\text { Paired comparison with baseline } \\
\text { p value }\end{array}$} \\
\hline & & & & & & & & 2 vs. 1 & 3 vs. 1 & 4 vs. 1 & 5 vs. 1 \\
\hline \multirow[t]{2}{*}{ Cortisol } & on drug & $0.28 \pm 0.29$ & $0.91 \pm 0.53$ & $2.19 \pm 1.15$ & $0.48 \pm 0.66$ & $0.36 \pm 0.46$ & $<0.001$ & $<0.001$ & $<0.001$ & 0.112 & 0.254 \\
\hline & off drug & $0.21 \pm 0.14$ & $0.25 \pm 0.18$ & $0.36 \pm 0.41$ & $0.38 \pm 0.37$ & $0.24 \pm 0.23$ & 0.366 & 0.563 & 0.197 & 0.160 & 0.678 \\
\hline \multirow[t]{2}{*}{ Testosterone } & on drug & $43.08 \pm 32.00$ & $71.33 \pm 47.66$ & $66.08 \pm 48.69$ & $43.17 \pm 22.57$ & $55.00 \pm 34.39$ & 0.075 & 0.004 & 0.041 & 0.991 & 0.299 \\
\hline & off drug & $47.75 \pm 43.40$ & $42.92 \pm 41.79$ & $42.33 \pm 34.23$ & $51.75 \pm 66.67$ & $40.75 \pm 28.50$ & 0.758 & 0.531 & 0.501 & 0.746 & 0.636 \\
\hline \multirow{2}{*}{$\begin{array}{l}\text { Body } \\
\text { temperature }\end{array}$} & on drug & $36.74 \pm 0.29$ & $36.75 \pm 0.32$ & $36.89 \pm 0.29$ & $36.73 \pm 0.18$ & $36.62 \pm 0.16$ & 0.233 & 0.956 & 0.324 & 0.930 & 0.082 \\
\hline & off drug & $36.83 \pm 0.25$ & $36.68 \pm 0.22$ & $36.64 \pm 0.32$ & $36.43 \pm 0.61$ & $36.50 \pm 0.68$ & 0.194 & 0.109 & 0.010 & 0.072 & 0.161 \\
\hline \multirow{2}{*}{$\begin{array}{l}\text { Ambient } \\
\text { temperature }\end{array}$} & on drug & $21.46 \pm 2.13$ & $22.67 \pm 1.67$ & $23.50 \pm 0.52$ & $22.33 \pm 1.23$ & $20.13 \pm 1.45$ & 0.001 & 0.16 & 0.007 & 0.255 & 0.160 \\
\hline & off drug & $21.75 \pm 2.68$ & $23.17 \pm 1.19$ & $23.17 \pm 1.03$ & $20.88 \pm 2.45$ & $20.75 \pm 1.77$ & 0.029 & 0.103 & 0.015 & 0.502 & 0.351 \\
\hline \multirow{2}{*}{$\begin{array}{l}\text { Feeling } \\
\text { hot }\end{array}$} & on drug & $2.08 \pm 1.17$ & $2.42 \pm 1.08$ & $3.75 \pm 0.87$ & $1.83 \pm 1.03$ & $1.92 \pm 1.00$ & $<0.001$ & 0.394 & $<0.001$ & 0.515 & 0.615 \\
\hline & off drug & $2.50 \pm 1.09$ & $2.50 \pm 1.17$ & $3.00 \pm 1.35$ & $2.00 \pm 0.85$ & $2.25 \pm 0.87$ & 0.239 & 1.000 & 0.377 & 0.139 & 0.571 \\
\hline \multirow{2}{*}{$\begin{array}{l}\text { Feeling } \\
\text { cold }\end{array}$} & on drug & $2.67 \pm 1.37$ & $2.33 \pm 1.23$ & $2.33 \pm 0.65$ & $2.08 \pm 1.31$ & $2.58 \pm 1.31$ & 0.373 & 0.474 & 0.305 & 0.111 & 0.674 \\
\hline & off drug & $2.75 \pm 1.42$ & $1.92 \pm 0.79$ & $1.58 \pm 0.67$ & $2.33 \pm 1.16$ & $2.50 \pm 1.38$ & 0.058 & 0.085 & 0.012 & 0.269 & 0.389 \\
\hline \multirow{2}{*}{$\begin{array}{l}\text { Hot/cold } \\
\text { flushes }\end{array}$} & on drug & $1.58 \pm 0.79$ & $2.42 \pm 1.08$ & $3.17 \pm 1.27$ & $1.33 \pm 0.89$ & $1.17 \pm 0.39$ & 0.001 & 0.075 & $<0.001$ & 0.515 & 0.096 \\
\hline & off drug & $1.58 \pm 1.00$ & $1.58 \pm 0.67$ & $1.25 \pm 0.45$ & $1.17 \pm 0.39$ & $1.00 \pm 0.00$ & 0.061 & 1.000 & 0.266 & 0.096 & 0.067 \\
\hline \multirow{2}{*}{$\begin{array}{l}\text { Feeling } \\
\text { thirsty }\end{array}$} & on drug & $3.08 \pm 0.67$ & $3.25 \pm 1.14$ & $3.67 \pm 1.07$ & $2.92 \pm 0.90$ & $3.17 \pm 0.94$ & 0.297 & 0.551 & 0.131 & 0.339 & 0.777 \\
\hline & off drug & $3.17 \pm 0.84$ & $3.08 \pm 1.24$ & $3.17 \pm 1.19$ & $2.92 \pm 1.08$ & $2.58 \pm 0.79$ & 0.295 & 0.674 & 1.000 & 0.389 & 0.089 \\
\hline \multirow[t]{2}{*}{ Sweating } & on drug & $1.58 \pm 0.79$ & $2.25 \pm 1.14$ & $3.50 \pm 1.00$ & $1.42 \pm 0.52$ & $1.25 \pm 0.45$ & $<0.001$ & 0.071 & $<0.001$ & 0.438 & 0.166 \\
\hline & off drug & $1.50 \pm 0.52$ & $1.83 \pm 0.84$ & $2.75 \pm 1.22$ & $1.42 \pm 0.67$ & $1.58 \pm 0.79$ & 0.005 & 0.339 & 0.003 & 0.723 & 0.754 \\
\hline
\end{tabular}

Each participant was assessed on self-administered MDMA and off MDMA, over counterbalanced weekends at the same club venue. Figures are group means \pm standard deviations. ANOVA session effect (Greenhouse-Geisser) and paired comparisons with pre-drug baseline are shown. Values represent degrees Celsius (body and ambient temperature), microgrammes/decilitre (cortisol), picogrammes/millilitre (testosterone) and 1-5 self-ratings (mood scales).

were discarded; this reduced the original sample size of 13 , to the 12 reported here). The agreement form stated that neither the University nor the experimenter condoned the use of illicit drugs and that the study should not be seen as providing approval or encouragement for the use of Ecstasy/MDMA or any other illegal drugs. The form also provided the contact details of the researcher. Website addresses and telephone numbers for drugs information lines were provided: www.talktofrank.com, www.substancemisuse.com and Frank telephone: 08007766 00. These provided a useful source of advice for drug-related problems and general drug issues. Throughout each test session, the data collectors maintained close links with the participants, in order to informally monitor their general well-being. No incidents arose which required their intervention. Following the study completion, informal feedback sessions were arranged.

\section{Statistical Analysis}

Each dependent variable was analyzed using 2-way repeated measures ANOVA with drug (2 levels) and test session (5 levels) as the 2 factors. Violations of sphericity were automatically adjusted using Greenhouse-Geisser corrections. The 2-way ANOVA was followed by a series of paired comparisons between the on- and off-MDMA conditions for each time point; values for $\mathrm{p}<0.01$ or above are highlighted in table 1 , in order to account for Bonfer- roni corrections (with 5 paired comparisons between the sessions). The on- and off-MDMA conditions were each analyzed separately by 1-way ANOVA, with test session or time as the repeated measures factor (tables 1 and 2). They were followed by paired comparison tests for difference scores for each post-drug test session compared to baseline, which were automatically corrected for multiple comparisons. Spearman rank-order correlations were performed between MDMA levels, cortisol values and the other dependent variables, for each dance club session. Testosterone correlations were not tested due to the mixed-gender sample. All the statistics were calculated using SPSS. All probability levels are 2tailed, with exact statistical significance levels being presented. The group data are shown as means and standard deviations.

\section{Results}

\section{Drug Usage}

The self-reported mean lifetime usage of Ecstasy/ MDMA was 56 occasions, with a range of 6-150. The mean duration of Ecstasy/MDMA use was 2.3 years, with 10 participants reporting $1-3$ years, the other 2 durations 
Table 2. Self-rated activities and mood states for 12 recreational Ecstasy/MDMA users before, during and after dance clubbing

\begin{tabular}{|c|c|c|c|c|c|c|c|c|c|c|c|}
\hline \multirow[t]{2}{*}{$\begin{array}{l}\text { Assessed } \\
\text { measure }\end{array}$} & \multirow[t]{2}{*}{$\begin{array}{l}\text { MDMA } \\
\text { status }\end{array}$} & \multirow[t]{2}{*}{$\begin{array}{l}\text { Before drug } \\
\text { (1) }\end{array}$} & \multirow{2}{*}{$\begin{array}{l}1 \mathrm{~h} \\
\text { after drug } \\
(2)\end{array}$} & \multirow{2}{*}{$\begin{array}{l}2.5 \mathrm{~h} \\
\text { after drug } \\
(3)\end{array}$} & \multirow{2}{*}{$\begin{array}{l}2 \text { days } \\
\text { after drug } \\
\text { (4) }\end{array}$} & \multirow{2}{*}{$\begin{array}{l}4 \text { days } \\
\text { after drug } \\
\text { (5) }\end{array}$} & \multirow{2}{*}{$\begin{array}{l}\text { p value } \\
\text { ANOVA } \\
\text { (session } \\
\text { effects) }\end{array}$} & \multicolumn{4}{|c|}{$\begin{array}{l}\text { Paired comparison with baseline } \\
\text { p value }\end{array}$} \\
\hline & & & & & & & & 2 vs. 1 & 3 vs. 1 & 4 vs. 1 & 5 vs. 1 \\
\hline \multirow{2}{*}{$\begin{array}{l}\text { Time } \\
\text { dancing }\end{array}$} & on drug & $1.25 \pm 0.62$ & $3.42 \pm 1.00$ & $4.33 \pm 0.49$ & $1.00 \pm 0.00$ & $1.08 \pm 0.29$ & $<0.001$ & $<0.001$ & $<0.001$ & 0.191 & 0.166 \\
\hline & off drug & $1.00 \pm 0.00$ & $2.83 \pm 0.94$ & $4.00 \pm 1.35$ & $1.08 \pm 0.29$ & $1.17 \pm 0.58$ & $<0.001$ & $<0.001$ & $<0.001$ & 0.339 & 0.339 \\
\hline \multirow{2}{*}{$\begin{array}{l}\text { Time } \\
\text { resting }\end{array}$} & on drug & $3.67 \pm 1.07$ & $2.58 \pm 1.17$ & $2.00 \pm 0.85$ & $4.08 \pm 0.90$ & $3.67 \pm 1.07$ & 0.001 & 0.059 & 0.006 & 0.175 & 1.000 \\
\hline & off drug & $3.42 \pm 1.17$ & $3.00 \pm 1.04$ & $2.00 \pm 1.21$ & $3.92 \pm 1.00$ & $3.58 \pm 1.00$ & $<0.001$ & 0.137 & 0.003 & 0.111 & 0.504 \\
\hline \multirow{2}{*}{$\begin{array}{l}\text { Time } \\
\text { drinking }\end{array}$} & on drug & $3.00 \pm 0.74$ & $2.92 \pm 0.90$ & $3.25 \pm 1.14$ & $2.67 \pm 1.07$ & $2.75 \pm 0.87$ & 0.564 & 0.820 & 0.586 & 0.457 & 0.491 \\
\hline & off drug & $2.42 \pm 0.79$ & $3.08 \pm 1.00$ & $2.50 \pm 1.17$ & $3.17 \pm 0.84$ & $2.75 \pm 1.06$ & 0.205 & 0.087 & 0.851 & 0.056 & 0.417 \\
\hline \multirow[t]{2}{*}{ Depressed } & on drug & $1.67 \pm 0.78$ & $1.08 \pm 0.29$ & $1.25 \pm 0.62$ & $1.83 \pm 0.84$ & $1.67 \pm 0.65$ & 0.022 & 0.027 & 0.137 & 0.586 & 1.000 \\
\hline & off drug & $1.75 \pm 0.87$ & $1.75 \pm 0.87$ & $1.75 \pm 1.06$ & $1.58 \pm 0.90$ & $1.67 \pm 0.78$ & 0.885 & 1.000 & 1.000 & 0.504 & 0.674 \\
\hline \multirow{2}{*}{$\begin{array}{l}\text { Quick- } \\
\text { witted }\end{array}$} & on drug & $2.92 \pm 0.90$ & $2.67 \pm 0.99$ & $2.67 \pm 1.07$ & $2.83 \pm 0.58$ & $2.58 \pm 0.90$ & 0.798 & 0.429 & 0.571 & 0.777 & 0.166 \\
\hline & off drug & $3.00 \pm 1.13$ & $3.00 \pm 0.95$ & $3.00 \pm 0.85$ & $3.17 \pm 0.94$ & $2.83 \pm 0.72$ & 0.742 & 1.000 & 1.000 & 0.586 & 0.504 \\
\hline \multirow[t]{2}{*}{ Excited } & on drug & $2.83 \pm 0.94$ & $3.75 \pm 0.87$ & $4.00 \pm 0.74$ & $2.25 \pm 0.75$ & $2.17 \pm 0.84$ & $<0.001$ & 0.034 & 0.001 & 0.067 & 0.013 \\
\hline & off drug & $2.50 \pm 0.67$ & $3.08 \pm 1.08$ & $3.33 \pm 1.16$ & $3.08 \pm 1.08$ & $2.67 \pm 1.16$ & 0.160 & 0.189 & 0.085 & 0.111 & 0.586 \\
\hline \multirow{2}{*}{$\begin{array}{l}\text { Socially } \\
\text { withdrawn }\end{array}$} & on drug & $1.50 \pm 0.67$ & $1.33 \pm 0.65$ & $1.33 \pm 0.65$ & $1.83 \pm 0.84$ & $2.00 \pm 0.95$ & 0.076 & 0.615 & 0.586 & 0.220 & 0.139 \\
\hline & off drug & $1.25 \pm 0.45$ & $1.67 \pm 0.65$ & $1.58 \pm 0.79$ & $1.75 \pm 0.97$ & $1.50 \pm 0.67$ & 0.398 & 0.054 & 0.166 & 0.166 & 0.275 \\
\hline \multirow[t]{2}{*}{ Anxiety } & on drug & $1.92 \pm 0.79$ & $1.58 \pm 0.67$ & $1.42 \pm 0.67$ & $1.67 \pm 0.89$ & $1.50 \pm 0.52$ & 0.403 & 0.368 & 0.191 & 0.429 & 0.137 \\
\hline & off drug & $2.00 \pm 1.21$ & $1.92 \pm 0.67$ & $1.67 \pm 0.49$ & $1.83 \pm 1.12$ & $2.08 \pm 1.17$ & 0.624 & 0.809 & 0.339 & 0.504 & 0.723 \\
\hline \multirow[t]{2}{*}{ Sociability } & on drug & $3.67 \pm 0.65$ & $4.33 \pm 0.65$ & $4.42 \pm 0.79$ & $3.42 \pm 0.79$ & $3.00 \pm 1.13$ & 0.002 & 0.039 & 0.056 & 0.389 & 0.120 \\
\hline & off drug & $3.75 \pm 1.06$ & $3.83 \pm 1.27$ & $3.83 \pm 1.27$ & $3.83 \pm 0.94$ & $3.00 \pm 0.74$ & 0.121 & 0.809 & 0.809 & 0.838 & 0.021 \\
\hline \multirow[t]{2}{*}{ Boredom } & on drug & $2.33 \pm 0.89$ & $1.50 \pm 0.67$ & $1.25 \pm 0.45$ & $2.33 \pm 0.89$ & $2.25 \pm 0.97$ & 0.004 & 0.054 & 0.003 & 1.000 & 0.845 \\
\hline & off drug & $2.75 \pm 1.06$ & $2.25 \pm 0.97$ & $2.50 \pm 1.09$ & $2.33 \pm 0.89$ & $2.67 \pm 1.30$ & 0.551 & 0.166 & 0.555 & 0.054 & 0.820 \\
\hline \multirow{2}{*}{$\begin{array}{l}\text { Clear- } \\
\text { headed }\end{array}$} & on drug & $3.58 \pm 1.08$ & $3.17 \pm 1.12$ & $2.92 \pm 1.00$ & $3.42 \pm 1.17$ & $3.00 \pm 1.28$ & 0.371 & 0.269 & 0.120 & 0.615 & 0.189 \\
\hline & off drug & $3.25 \pm 1.14$ & $3.83 \pm 1.03$ & $4.08 \pm 1.08$ & $3.50 \pm 1.17$ & $3.67 \pm 0.78$ & 0.054 & 0.131 & 0.005 & 0.191 & 0.137 \\
\hline \multirow[t]{2}{*}{ Relaxed } & on drug & $3.92 \pm 0.67$ & $3.92 \pm 1.17$ & $4.17 \pm 1.19$ & $3.92 \pm 0.90$ & $3.58 \pm 0.90$ & 0.590 & 1.000 & 0.536 & 1.000 & 0.266 \\
\hline & off drug & $3.92 \pm 0.79$ & $3.67 \pm 1.07$ & $4.17 \pm 0.72$ & $3.83 \pm 1.19$ & $3.75 \pm 1.22$ & 0.610 & 0.586 & 0.463 & 0.754 & 0.658 \\
\hline \multirow[t]{2}{*}{ Unpleasant } & on drug & $1.33 \pm 0.49$ & $1.33 \pm 0.65$ & $1.17 \pm 0.39$ & $1.83 \pm 0.58$ & $1.75 \pm 0.87$ & 0.082 & 1.000 & 0.339 & 0.053 & 0.210 \\
\hline & off drug & $1.67 \pm 0.65$ & $1.67 \pm 0.65$ & $1.75 \pm 1.14$ & $1.67 \pm 0.65$ & $1.58 \pm 0.79$ & 0.946 & 1.000 & 0.838 & 1.000 & 0.674 \\
\hline \multirow[t]{2}{*}{ Happiness } & on drug & $3.67 \pm 0.65$ & $4.00 \pm 1.04$ & $4.42 \pm 0.52$ & $3.67 \pm 0.65$ & $3.17 \pm 0.84$ & 0.012 & 0.220 & 0.005 & 1.000 & 0.166 \\
\hline & off drug & $3.75 \pm 0.87$ & $3.92 \pm 0.90$ & $3.83 \pm 0.94$ & $3.67 \pm 0.78$ & $3.58 \pm 0.67$ & 0.710 & 0.658 & 0.838 & 0.754 & 0.438 \\
\hline \multirow[t]{2}{*}{ Tired } & on drug & $2.50 \pm 0.52$ & $1.50 \pm 0.80$ & $2.25 \pm 1.14$ & $3.17 \pm 0.94$ & $3.75 \pm 0.75$ & $<0.001$ & 0.004 & 0.389 & 0.025 & $<0.001$ \\
\hline & off drug & $2.83 \pm 1.03$ & $2.42 \pm 1.00$ & $3.08 \pm 0.90$ & $2.67 \pm 0.89$ & $2.75 \pm 0.75$ & 0.281 & 0.269 & 0.463 & 0.615 & 0.809 \\
\hline Impaired & on drug & $2.17 \pm 0.94$ & $2.67 \pm 1.30$ & $2.67 \pm 1.30$ & $2.83 \pm 0.84$ & $2.58 \pm 1.31$ & 0.501 & 0.256 & 0.256 & 0.136 & 0.318 \\
\hline concentration & off drug & $2.67 \pm 1.07$ & $2.17 \pm 1.27$ & $2.00 \pm 1.13$ & $2.25 \pm 1.06$ & $2.50 \pm 1.00$ & 0.146 & 0.111 & 0.005 & 0.096 & 0.586 \\
\hline Energy & on drug & $2.58 \pm 1.00$ & $3.58 \pm 1.08$ & $4.00 \pm 0.74$ & $2.08 \pm 0.67$ & $2.08 \pm 0.90$ & $<0.001$ & 0.053 & 0.002 & 0.139 & 0.053 \\
\hline & off drug & $2.33 \pm 0.78$ & $3.08 \pm 1.00$ & $3.25 \pm 1.06$ & $2.83 \pm 1.03$ & $2.33 \pm 0.89$ & 0.076 & 0.095 & 0.050 & 0.082 & 1.000 \\
\hline Memory & on drug & $1.92 \pm 0.90$ & $1.50 \pm 0.80$ & $2.00 \pm 0.95$ & $2.25 \pm 1.22$ & $2.42 \pm 1.24$ & 0.176 & 0.241 & 0.809 & 0.368 & 0.191 \\
\hline & off drug & $2.58 \pm 1.08$ & $2.33 \pm 1.44$ & $1.92 \pm 1.31$ & $2.17 \pm 1.12$ & $2.08 \pm 0.79$ & 0.260 & 0.389 & 0.013 & 0.175 & 0.053 \\
\hline
\end{tabular}

Each participant was assessed on self-administered MDMA and off MDMA, over counterbalanced weekends at the same club venue. Figures are group means \pm standard deviations. ANOVA session effect (Greenhouse-Geisser) and paired comparisons with pre-drug baseline are shown. Values represent $1-5$ self-ratings (mood scales).

being 6 months and 10 years. The average number of tablets usually taken per occasion was 2.3 , with a range of 1-3. The largest number of ecstasy tablets on 1 occasion ranged from 2 tablets for the most novice user to 10 for the most experienced lifetime user; the median being 5 tablets. In terms of other drug usage, 10 participants had previously taken cocaine (mean $=26.2$ occasions for the users), 11 had used psilocybin 'magic' mushrooms 
Table 3. Summary of 2-way ANOVA findings (Greenhouse-Geisser corrections)

\begin{tabular}{|c|c|c|c|c|c|c|c|c|}
\hline \multirow[t]{2}{*}{ Measure } & \multicolumn{3}{|c|}{ 2-way ANOVA } & \multicolumn{5}{|c|}{ Paired $t$ test comparison between MDMA and abstinence weekend } \\
\hline & $\begin{array}{l}\text { drug } \\
(\text { d.f. }=1)\end{array}$ & $\begin{array}{l}\text { time } \\
(\text { d.f. }=4)\end{array}$ & $\begin{array}{l}\text { drug } \times \text { time } \\
\text { interaction } \\
(\text { d.f. }=4)\end{array}$ & $\begin{array}{l}\text { before } \\
\text { drug } \\
(1)\end{array}$ & $\begin{array}{l}1 \mathrm{~h} \\
\text { after drug } \\
\text { (2) }\end{array}$ & $\begin{array}{l}2.5 \mathrm{~h} \\
\text { after drug } \\
\text { (3) }\end{array}$ & $\begin{array}{l}2 \text { days } \\
\text { after drug } \\
\text { (4) }\end{array}$ & $\begin{array}{l}4 \text { days } \\
\text { after drug } \\
\text { (5) }\end{array}$ \\
\hline Cortisol & $<0.001$ & $<0.001$ & $<0.001$ & 0.341 & $<0.001$ & $<0.001$ & 0.619 & 0.130 \\
\hline Testosterone & 0.075 & 0.543 & 0.083 & 0.537 & 0.008 & 0.066 & 0.608 & 0.069 \\
\hline Body temperature & 0.170 & 0.198 & 0.202 & 0.408 & 0.588 & 0.088 & 0.085 & 0.505 \\
\hline Ambient temperature & 0.682 & 0.001 & 0.287 & 0.758 & 0.410 & 0.104 & 0.110 & 0.394 \\
\hline Steps completed & 0.619 & $\mathrm{n} / \mathrm{a}$ & $\mathrm{n} / \mathrm{a}$ & $\mathrm{n} / \mathrm{a}$ & $\mathrm{n} / \mathrm{a}$ & $\mathrm{n} / \mathrm{a}$ & $\mathrm{n} / \mathrm{a}$ & $\mathrm{n} / \mathrm{a}$ \\
\hline Time dancing & 0.118 & $<0.001$ & 0.240 & 0.191 & 0.012 & 0.438 & 0.339 & 0.674 \\
\hline Time resting & 0.923 & $<0.001$ & 0.623 & 0.463 & 0.210 & 1.000 & 0.551 & 0.820 \\
\hline Time drinking & 0.232 & 0.749 & 0.135 & 0.089 & 0.615 & 0.095 & 0.236 & 1.000 \\
\hline Depressed & 0.397 & 0.384 & 0.099 & 0.754 & 0.025 & 0.214 & 0.536 & 1.000 \\
\hline Quick-witted & 0.039 & 0.606 & 0.945 & 0.795 & 0.266 & 0.266 & 0.368 & 0.389 \\
\hline Excited & 0.779 & $<0.001$ & 0.011 & 0.305 & 0.136 & 0.120 & 0.010 & 0.214 \\
\hline Socially withdrawn & 0.803 & 0.146 & 0.228 & 0.339 & 0.266 & 0.463 & 0.845 & 0.111 \\
\hline Anxiety & 0.209 & 0.233 & 0.773 & 0.857 & 0.220 & 0.389 & 0.701 & 0.152 \\
\hline Sociability & 0.610 & 0.002 & 0.202 & 0.845 & 0.166 & 0.171 & 0.295 & 1.000 \\
\hline Boredom & 0.014 & 0.014 & 0.157 & 0.339 & 0.056 & 0.006 & 1.000 & 0.269 \\
\hline Clear headed & 0.008 & 0.795 & 0.076 & 0.368 & 0.054 & 0.012 & 0.845 & 0.054 \\
\hline Relaxed & 0.883 & 0.425 & 0.872 & 1.000 & 0.536 & 1.000 & 0.866 & 0.701 \\
\hline Unpleasant & 0.144 & 0.541 & 0.209 & 0.039 & 0.220 & 0.111 & 0.586 & 0.504 \\
\hline Happiness & 0.799 & 0.019 & 0.262 & 0.777 & 0.851 & 0.046 & 1.000 & 0.096 \\
\hline Tired & 0.668 & $<0.001$ & 0.002 & 0.417 & 0.020 & 0.117 & 0.275 & 0.020 \\
\hline Impaired concentration & 0.235 & 0.837 & 0.099 & 0.166 & 0.214 & 0.071 & 0.152 & 0.820 \\
\hline Energy & 0.555 & 0.002 & 0.006 & 0.191 & 0.053 & 0.069 & 0.043 & 0.463 \\
\hline Memory & 0.362 & 0.448 & 0.088 & 0.025 & 0.107 & 0.862 & 0.674 & 0.305 \\
\hline Hotness & 0.817 & 0.001 & 0.220 & 0.339 & 0.838 & 0.145 & 0.638 & 0.339 \\
\hline Coldness & 0.308 & 0.115 & 0.168 & 0.809 & 0.210 & 0.021 & 0.491 & 0.777 \\
\hline Flushes & 0.001 & $<0.001$ & 0.003 & 1.000 & 0.044 & 0.001 & 0.438 & 0.166 \\
\hline Sweating & 0.394 & $<0.001$ & 0.182 & 0.777 & 0.339 & 0.169 & 1.000 & 0.166 \\
\hline Thirst & 0.290 & 0.230 & 0.406 & 0.754 & 0.551 & 0.256 & 1.000 & 0.189 \\
\hline
\end{tabular}

Drug (2) = On and off MDMA; time/session (5) = pre-drug baseline, 1 and $2.5 \mathrm{~h}$ after drug while dance clubbing, 2 and 4 days after drug. Significance of within-subject effects given as a p value (level of significance indicated in italics); for the paired comparison, $<1 \%$ is considered significant to account for the Bonferroni correction. $\mathrm{n}=12$ in each case. The number of steps completed was over the total time using a pedometer and therefore, the significance was measured with a paired $t$ test.

(mean $=8.3$ occasions for users), 11 had utilized amyl nitrite inhalers or 'poppers' (mean $=18.1$ occasions for users), and 7 had previously taken amphetamine (mean $=$ 6.6 occasions for users). None of the group reported having ever used opiates, $\gamma$-hydroxybutyrate, solvents or anabolic steroids. Three participants reported having utilized ketamine (on 1, 5 and $>5$ occasions); 3 had used benzodiazepines/barbiturates (on 3, 10 and $>10$ occasions), and 2 had taken LSD ( 1 and $>10$ times). Eight of the sample were tobacco smokers (mean $=48$ cigarettes/ week; range $=4-100$ ). Cannabis was consumed by 11 of the participants, generally at moderate levels (i.e. occasional/regular rather than heavy/dependent smoking).
Every participant reported alcohol drinking, with the 4 females indicating an average of 17 units of alcohol/week (range $=10-28$ ), and the 8 males mentioned an average of 46 units of alcohol/week (range $=10-70$ ).

The saliva analyses showed that all 12 participants who signed up for the study were free from MDMA when dance clubbing during abstinence, since all values were 0 at both the 1- and 2.5-hour (off-MDMA) sessions. The values were also zero 2 and 4 days later. The saliva tests confirmed that all 12 participants were positive for MDMA during the on-Ecstasy dance club sessions. At the 1-hour session, the group mean MDMA value was $1,524 \mathrm{ng} / \mathrm{ml}$ (range $=74-7,025)$, while at the 2.5 -hour ses- 

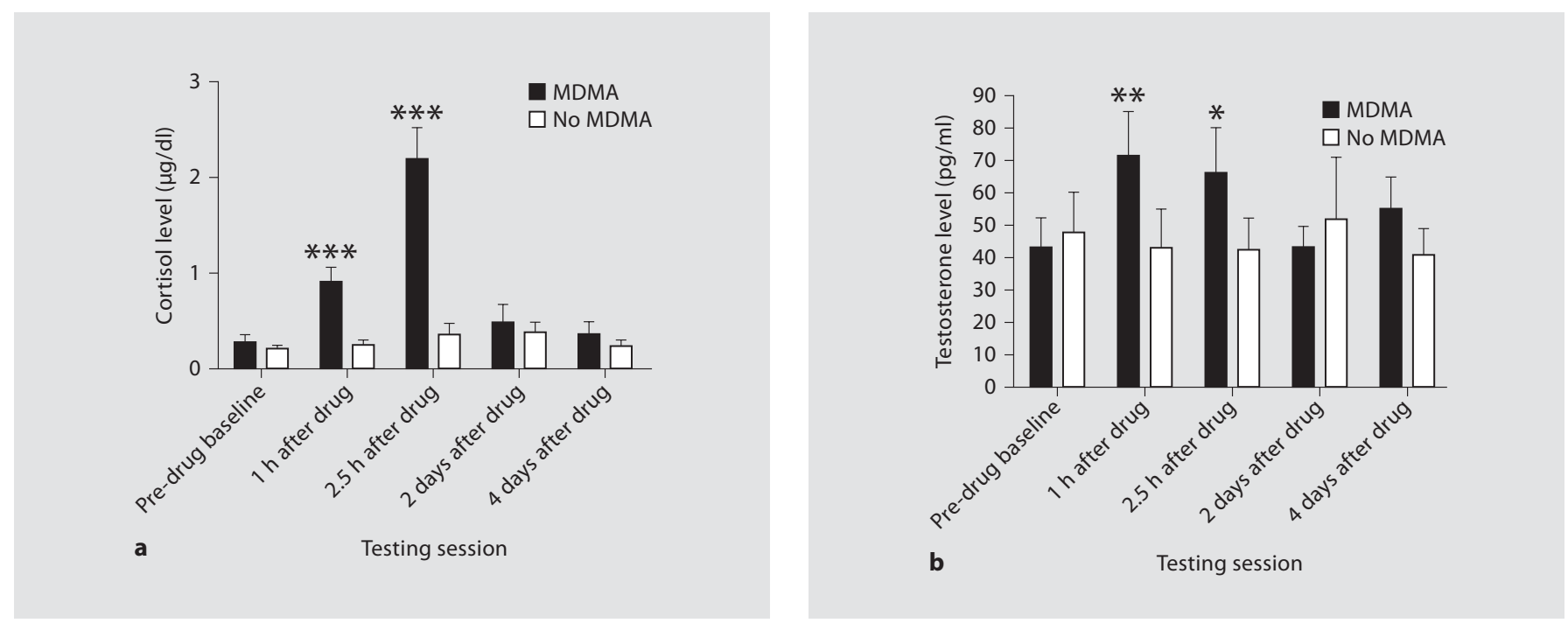

Fig. 1. Cortisol (a) and testosterone (b) levels for 12 recreational Ecstasy/MDMA users before, during and after dance clubbing. Each participant was assessed on self-administered MDMA and off MDMA over counterbalanced weekends at the same dance club. Significance levels are comparisons with pre-drug baseline. Means and standard error bars. Times are approximate with a $10 \%$ variability. ${ }^{*} \mathrm{p}<0.05 ;{ }^{* *} \mathrm{p}<0.01 ;{ }^{* *} \mathrm{p}<0.001$.

sion, it was $3,447 \mathrm{ng} / \mathrm{ml}$ (range $=396-17,166)$. The preMDMA baseline values were all 0 , but 3 of the day 2 values were positive, although at very low levels $(34,55$ and $138 \mathrm{ng} / \mathrm{ml}$ ), and 2 of the day 4 values were also positive, 1 at a higher level (138 and 5,254 $\mathrm{ng} / \mathrm{ml})$. This latter value was similar to the one for that participant 4 days earlier $2.5 \mathrm{~h}$ after taking MDMA (5,681 $\mathrm{ng} / \mathrm{ml})$, and suggests that Ecstasy/MDMA may have been taken on day 4. This particular participant also showed a residual value for the pre-abstinence baseline 3 days later $(24 \mathrm{ng} / \mathrm{ml})$, although all the other abstinence condition values were 0 . Since this participant was free from MDMA when dance clubbing during the abstinence condition, the data were retained within the study.

\section{Neuroendocrine Measures}

The cortisol and testosterone findings are presented in tables 1 and 3 and figure 1. The cortisol levels demonstrated a significant 2 -way ANOVA drug effect $(\mathrm{p}<$ $0.001)$, session effect $(p<0.001)$ and drug by session interaction $(\mathrm{p}<0.001$; table 3$)$. Post-hoc comparison tests revealed that the cortisol levels differed significantly between the on- and off-MDMA conditions, at the 1- ( $p<$ $0.001)$ and the 2.5-hour post-drug sessions $(\mathrm{p}<0.001$; table 3 ). These differences between the drug conditions reflected the very strong rise in cortisol following MDMA, with a significant increase $1 \mathrm{~h}$ after MDMA ( $\mathrm{p}<$
$0.001)$ and $2.5 \mathrm{~h}$ after MDMA ( $<<0.001$; table 1$)$. These values indicated increases over baseline of around 300\% at $1 \mathrm{~h}$ and around $800 \%$ after $2.5 \mathrm{~h}$ on MDMA (fig. 1, table 1). In the MDMA abstinence condition, the cortisol values did not change significantly over time, although the mean cortisol value increased by around 70\%, (table 1; fig. 1). The testosterone levels showed no significant 2 -way ANOVA effects, although drug $(\mathrm{p}<0.10)$ and time $(p<0.10)$ were statistically borderline. Post-hoc comparison tests revealed significantly higher testosterone $1 \mathrm{~h}$ after MDMA compared to MDMA abstinence $(\mathrm{p}=0.008)$, while the difference in testosterone at the 2.5 -hour session was statistically borderline $(\mathrm{p}=0.066$; table 3$)$. The 1-way ANOVA session effect for testosterone was borderline for the MDMA condition ( $\mathrm{p}=0.075)$. In the paired comparisons with baseline, there were significant increases in testosterone $1 \mathrm{~h}$ after MDMA ( $\mathrm{p}=0.004)$ and $2.5 \mathrm{~h}$ after MDMA ( $\mathrm{p}=0.041)$; these values reflected percentage increases of around 80 and $60 \%$ respectively.

\section{Thermal Aspects}

The objective and subjective thermal assessment measures are presented in tables 1 and 3 and figure 2. Body temperature showed a slight tendency to rise after taking MDMA and to fall slightly in the abstinence condition (fig. 2, table 1). The group mean temperature increase of $0.15^{\circ} \mathrm{C}$ after $2.5 \mathrm{~h}$ on MDMA was non-significant, where- 


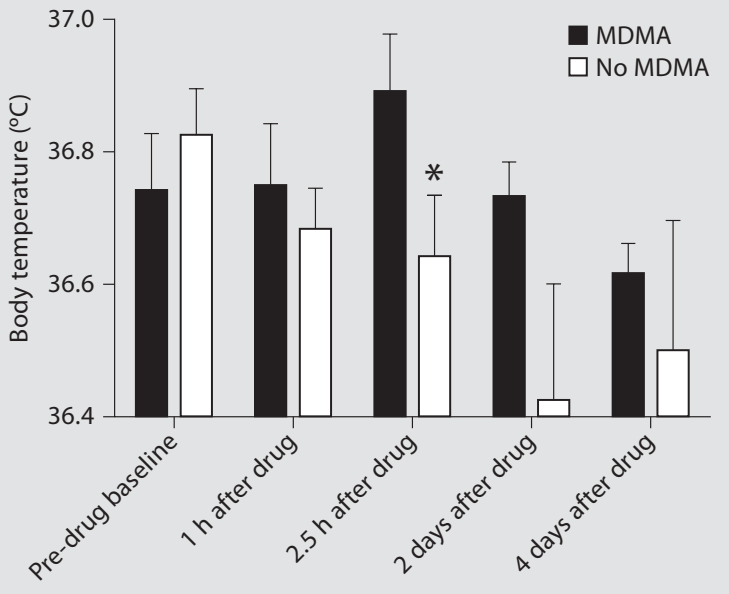

Testing session

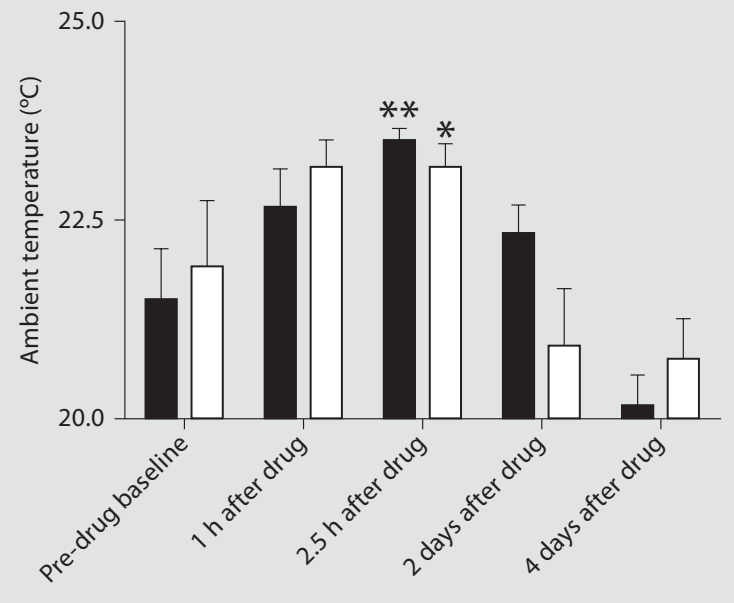

Testing session
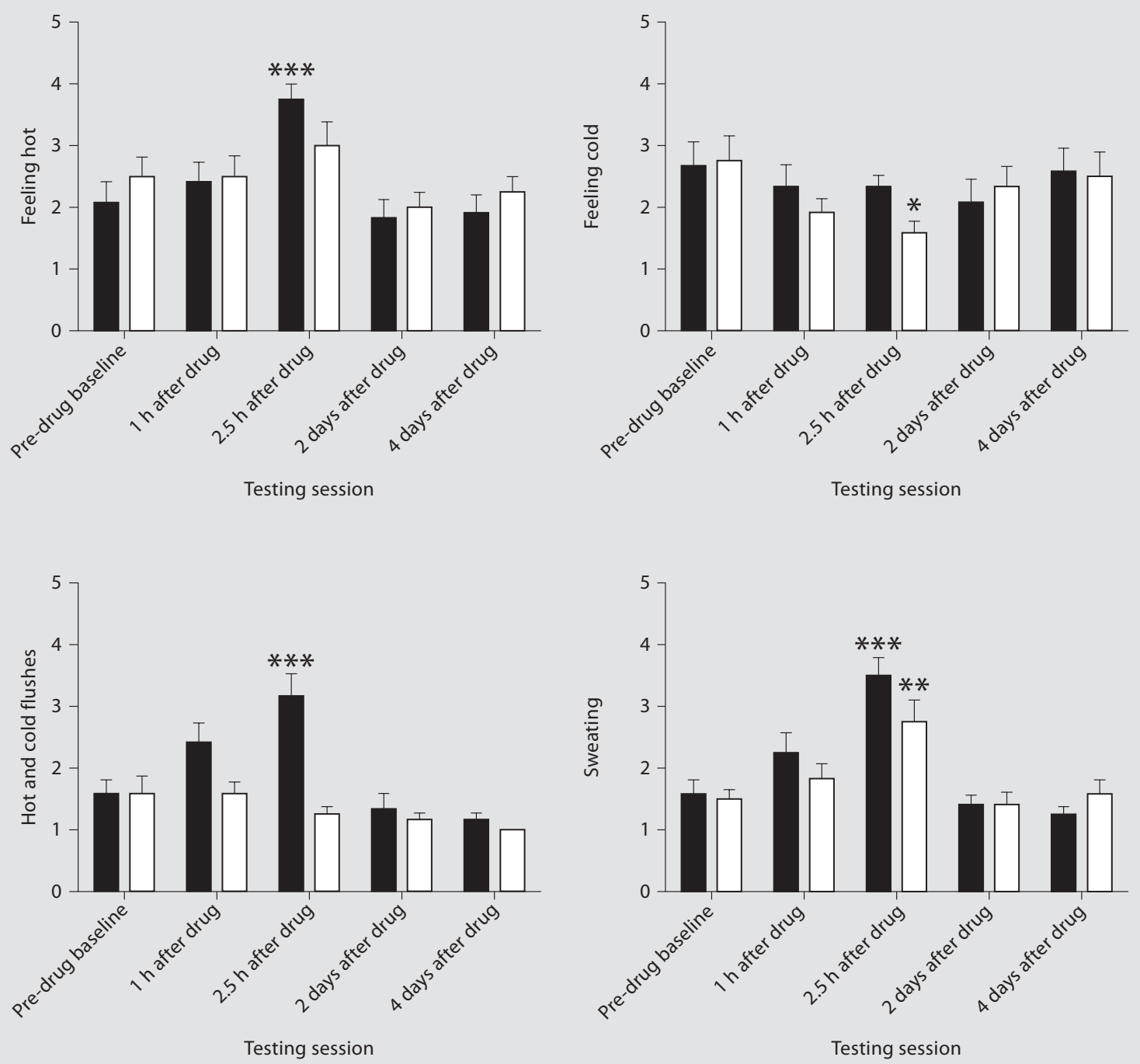
as the decrease of $0.19^{\circ} \mathrm{C}$ after $2.5 \mathrm{~h}$ of abstinence from MDMA was significant ( $<<0.010$, table 1$)$. The paired comparison test between the on- and off-MDMA conditions, at the 2.5-hour clubbing session, was statistically borderline ( $p=0.088$; table 3$)$. However these change scores partially reflect the higher baseline values for the abstinence condition (fig. 2, table 1). Ambient temperature showed a significant 2-way ANOVA session effect $(\mathrm{p}<0.001)$. This was also evident in the 1-way ANOVAs, where session was significant for both the on- $(\mathrm{p}<0.001)$ and the off-MDMA conditions ( $\mathrm{p}=0.029)$. These values reflected the significant increase in ambient temperature at the second dance club session, under both drug conditions (table 1, fig. 2).

Hot and cold flushes demonstrated a significant 2-way ANOVA drug $(\mathrm{p}<0.001)$, session $(\mathrm{p}<0.001)$ and drug by session interaction effect $(p<0.003$; table 3$)$. This reflected the significantly higher levels of hot and cold flushes $1 \mathrm{~h}$ after MDMA ( $\mathrm{p}=0.044)$ and $2.5 \mathrm{~h}$ after MDMA ( $<<0.001$; table 3). Figure 2 shows that there were no changes in hot and cold flushes in the MDMA abstinence condition. The significant effect of acute MDMA on hot and cold flushes was confirmed in the paired comparisons with baseline. Here there was a borderline increase in hot and cold flushes $1 \mathrm{~h}$ after MDMA ( $<<0.075)$ and a significant increase $2.5 \mathrm{~h}$ after MDMA $(\mathrm{p}<0.001)$ but no equivalent increases when off MDMA (table 1). The self-rated levels of feeling hot showed a significant 2 -way ANOVA session effect $(\mathrm{p}<$ 0.001; table 3). The 1-way ANOVA for the on-MDMA condition also revealed a significant session effect $(p<0.001)$, with the paired comparisons indicating that this was due to a significant increase in feeling hot $2.5 \mathrm{~h}$ after MDMA self-administration ( $\mathrm{p}<0.001$; fig. 2 ; table 1 ). The self-rated levels of feeling cold showed no significant ANOVA effects. However post-hoc tests found that feeling cold was significantly reduced under MDMA abstinence at the 2.5hour clubbing session (fig. 2; table 1). Feeling thirsty demonstrated no significant ANOVA effects (tables 1 and 3). The self-rated levels of sweating showed significant session effects, in the 2-way ( $\mathrm{p}<0.001$; table 3$)$ and the 1-way ANOVAs for both the on- $(\mathrm{p}<0.001)$ and off-MDMA con-

Fig. 2. Body temperature and thermal self-ratings for 12 recreational Ecstasy/MDMA users before, during and after dance clubbing. Each participant was assessed on self-administered MDMA and off MDMA over counterbalanced weekends at the same dance club. Significance levels are comparisons with predrug baseline. Means and standard error bars. Times are approximate with a $10 \%$ variability. ${ }^{*} \mathrm{p}<0.05 ;{ }^{* *} \mathrm{p}<0.01$; ${ }^{* * *} \mathrm{p}<$ 0.001 .

Dance Clubbing on MDMA ditions ( $p<0.005$; table 1 ). Post-hoc tests revealed that these reflected an increase in sweating at the 2.5-hour clubbing session, when on $(\mathrm{p}<0.001)$ and off MDMA $(\mathrm{p}=$ 0.003; table 1).

\section{Physical Activity}

The pedometer scores indicated that there was no differences in physical activity between the on- and offMDMA dance club sessions ( $\mathrm{p}=0.619$; table 3 ). The selfrated levels of dancing showed a significant 2-way ANOVA session effect ( $\mathrm{p}<0.001$; table 3$)$. A significant session effect was also evident in the 1-way ANOVAs, both for the on- $(\mathrm{p}<0.001)$ and the off-MDMA conditions $(\mathrm{p}<$ 0.001 ; table 2). The paired comparison tests showed that this reflected a significant increase in dancing at the 1and 2.5-hour dance clubbing sessions, for both the onand off-MDMA conditions (all values $\mathrm{p}<0.001$; table 2 ). An opposite pattern was found for the self-rated levels of resting, with significant 1- and 2-way ANOVA session effects (all p < 0.001) and significant reductions in the amount of time spent resting, at both of the dance club sessions, whether on or off MDMA (tables 2 and 3).

\section{Mood States}

The ANOVA mood state findings are presented in tables 2 and 3. Significant 2-way ANOVA session effects were present for feelings of excitement $(\mathrm{p}<0.001)$, energy $(\mathrm{p}<0.002)$, tiredness $(\mathrm{p}<0.001)$, boredom $(\mathrm{p}<0.014)$, sociability $(\mathrm{p}<0.002)$ and happiness $(\mathrm{p}<0.019$; table 3$)$. Significant 2-way ANOVA drug effects were shown for feelings of clearheadedness $(\mathrm{p}<0.008)$, quick-wittedness $(\mathrm{p}<0.039)$ and boredom ( $\mathrm{p}<0.014$; table 3$)$. The drug by session interaction was significant for feelings of energy $(\mathrm{p}<0.006)$, tiredness $(\mathrm{p}<0.002)$ and excitement ( $\mathrm{p}<$ 0.011 ; table 3 ). The general pattern was for mood improvements at the club after MDMA compared to baseline. Hence after MDMA there were significant increases in self-rated excitement ( 1 and $2.5 \mathrm{~h}$ ), lessened feelings of depression $(1 \mathrm{~h})$, reduced tiredness $(1 \mathrm{~h})$, greater sociability $(1 \mathrm{~h})$, reduced boredom $(2.5 \mathrm{~h})$, greater happiness $(2.5 \mathrm{~h})$ and greater energy ( $2.5 \mathrm{~h}$; table 2$)$. In the MDMA abstinence condition, there were 3 significant changes compared to baseline, with greater clearheadedness and reduced levels of memory problems and concentration difficulties (all $2.5 \mathrm{~h}$; table 2). Turning to the mood state comparisons between the on- and off-MDMA conditions at the 2 clubbing sessions, most of these were non-significant, with moods generally improving under both drug conditions (table 3), although there were significant differences in feelings of depression and boredom. Self-rated 
Table 4. Psychobiological health changes attributed to recreational MDMA: participants listed according to lifetime Ecstasy usage

\begin{tabular}{ll}
\hline Lifetime usage/gender & $\begin{array}{l}\text { Psychobiological/health changes attrib- } \\
\text { uted to Ecstasy/MDMA }\end{array}$
\end{tabular}

\begin{tabular}{|c|c|}
\hline 6 occasions/female & $\begin{array}{l}\text { After a very heavy(ish) month, experi- } \\
\text { enced very heavy/strong pre-menstrual } \\
\text { tension - depressed, moody and angry, } \\
\text { much more than normal }\end{array}$ \\
\hline 8 occasions/male & $\begin{array}{l}\text { Mood fluctuations and anxiety } 1-2 \text { days } \\
\text { after; tired for } 1 \text { week }\end{array}$ \\
\hline 15 occasions/female & $\begin{array}{l}\text { Short-term mild depression for } 2-3 \text { days } \\
\text { after taking Ecstasy; worth it }\end{array}$ \\
\hline 15 occasions/male & $\begin{array}{l}\text { Do not feel } 100 \% \text { next day, quite tired, } \\
\text { watched a lot of TV }\end{array}$ \\
\hline 15 occasions/male & $\begin{array}{l}\text { Twitches/brain flashes a few days after } \\
\text { use }\end{array}$ \\
\hline 20 occasions/female & $\begin{array}{l}\text { Mood swings, memory problems, disor- } \\
\text { dered memory }\end{array}$ \\
\hline$\geq 20$ occasions/male & Slight depression next day \\
\hline 50 occasions/male & $\begin{array}{l}\text { Chapped lips; memory problems; mild } \\
\text { hallucinations after sustained use }\end{array}$ \\
\hline 80 occasior & $\begin{array}{l}\text { Occasional - memory, depressed, mood } \\
\text { fluctuations, loss of sleep, headache, loss } \\
\text { of appetite, stomach ache - up to } 4 \text { days } \\
\text { after event }\end{array}$ \\
\hline 150 occasions/male & $\begin{array}{l}\text { No health problems; weight loss, detesta- } \\
\text { tion of food in the immediate period fol- } \\
\text { lowing (and in anticipation of) nights on } \\
\text { Ecstasy; despite feeling mellow the day } \\
\text { after, can have depressed moods } 2 \text { days } \\
\text { or more after for 1-2 days; not sure, } \\
\text { but memory loss has been remarked by } \\
\text { friends and workmates }\end{array}$ \\
\hline 150 occasions/male & $\begin{array}{l}\text { Major memory problems; feel pretty } \\
\text { dosile (sic) on a come-down }\end{array}$ \\
\hline 150 occasions/male & $\begin{array}{l}\text { Mood problems up to a week later - de- } \\
\text { pression; crap memory }\end{array}$ \\
\hline
\end{tabular}

depression was significantly lower $1 \mathrm{~h}$ after MDMA compared to abstinence $(\mathrm{p}=0.025)$, while self-rated boredom was significantly lower $2.5 \mathrm{~h}$ after MDMA compared to abstinence ( $\mathrm{p}=0.006$; table 3 ). Turning to the recovery period, the self-rated levels of tiredness were significantly higher $2(\mathrm{p}<0.025)$ and 4 days after MDMA $(\mathrm{p}<0.001)$. Feelings of excitement were significantly reduced 4 days after MDMA ( $<<0.013)$. Self-rated sociability was re- duced 4 days after the abstinence session $(\mathrm{p}<0.021)$, although it should be noted that the mean sociability score was identical to the equivalent post-MDMA value (the onMDMA value showed greater variance, and this will have contributed to its lower significance; table 2).

\section{Spearman Correlations}

The cortisol levels correlated significantly with selfreported hot and cold flushes at the first dance club session $(\mathrm{r}=+0.88, \mathrm{p}=0.001)$, while cortisol was also positively associated with the time spent drinking at this first session $(\mathrm{r}=+0.59, \mathrm{p}=0.044)$; the time spent drinking also correlated positively with hot and cold flushes $(r=+0.57$, $\mathrm{p}=0.051)$ and with 'feeling thirsty' $(\mathrm{r}=+0.77, \mathrm{p}=0.003)$. This cluster of positive inter-correlations was however not repeated at the second dance club session, since none of the relationships between cortisol and the other dependent variables was significant. The MDMA saliva levels at the first dance club session were positively associated with feeling thirsty $(\mathrm{r}=+0.63, \mathrm{p}=0.028)$. MDMA at the second dance club session correlated inversely with feeling anxious $(\mathrm{r}=-0.72, \mathrm{p}=0.008)$ and hot and cold flushes $(r=-0.68, p=0.016)$. None of the other MDMA or cortisol associations was significant.

\section{Health and Psychobiological Changes}

Table 4 lists the adverse health and other psychobiological changes attributed to Ecstasy/MDMA. Every participant noted at least 1 problem, although their nature tended to alter in parallel with lifetime usage. Those who had taken MDMA on less than 20 occasions complained of mood swings and tiredness in the post-MDMA recovery period. One female noted that her premenstrual tension was exacerbated, whereas another of the novice users stated that the mild depression which followed MDMA was 'worth it'. Poor memory was not noted by any of the 5 novice users, whereas 6 of the 7 more experienced users (20 or more lifetime occasions) complained of memory problems (table 4). Other psychobiological difficulties mentioned by these more experienced users included depressed mood states, loss of sleep and food/appetite issues.

\section{Discussion}

\section{Original Design Aspects}

This is the first study to empirically compare dance clubbers when they have taken MDMA, with the same clubbers over a weekend of MDMA abstinence. The im- 
portance of this design is that it allowed the environmental aspects which surround recreational ecstasy use to be held constant, while varying the central factor of MDMA usage. Furthermore under the abstinence condition, each participant danced at their normal club venue, with the same group of friends. The levels of dancing each week were also very similar, as measured by both self-report and pedometer (table 3). The aim of keeping social and environmental factors constant across conditions was therefore largely achieved. The biochemical analyses also confirmed that every participant was on MDMA during the on-drug weekend, and that they were free from MDMA while clubbing during abstinence. The ELISA saliva sample analysis procedures we used have been demonstrated to be highly sensitive. In a laboratory study, Laloup et al. (2005) reported a sensitivity of $98.6 \%$ for ELISA saliva analyses from human volunteers given acute doses of 75 and $100 \mathrm{mg}$ of MDMA.

\section{Neuroendocrine Changes}

The cortisol levels showed a highly significant increase of $800 \%$ following Ecstasy/MDMA, compared to predrug baseline values (table $1 ; \mathrm{p}<0.001$, fig. 1 ). This is the first study to investigate cortisol in dance using clubbers, and the extent of this cortisol increase was much greater than expected. Laboratory studies have found a significant increase in cortisol following acute MDMA [23-28, 39], but the maximal percentage increase they reported was around $150 \%$. One possible reason for the far larger rise observed in the present study was that the drug was being taken by physically active dancers. The self-rated levels of dancing were significantly increased at both dance club sessions, when on and off MDMA (table 1), and the non-significant increase in cortisol of $70 \%$ in our abstinent dance clubbers may reflect this physical activity (fig. 1). One of the primary roles of the HPA axis is to prepare the body for physical action so that cortisol is often characterized as a 'general activity' hormone [47]. Cortisol is thus released during sports and other physical activities, and the degree of increase correlates with the intensity and duration of exercise [48, 49], although we have not found any study reporting an acute cortisol increase which approaches $800 \%$. For instance, Davis et al. [48] monitored 12 experienced and 7 naïve young healthy males who cycled 'to exhaustion' on a laboratory ergometer. After undergoing this extreme physical stressor the experienced subjects demonstrated a $59 \%$ increase in cortisol, whereas the naïve volunteers showed a $138 \%$ rise. In a unique study of students at a 'pre-Ecstasy' disco dance club, with loud music and bright psychedelic lights

Dance Clubbing on MDMA for around $5 \mathrm{~h}$ : 'an increase in urinary excretion of cortisol ... was observed in most of the subjects during their presence in the discotheque' [84]; this possibly suggests a similar magnitude of neuroendocrine change to the abstinence condition here.

The large $800 \%$ cortisol rise in our dance clubbers when on MDMA (fig. 1) may therefore reflect the combined or interactive effects of drug stimulation and physical activity. This hypothesis needs to be further investigated. In particular, future studies should focus on the cortisol effects of MDMA in active dance clubbers versus more sedentary users. We have confirmed an $800 \%$ cortisol increase in a more recent study of recreational Ecstasy/MDMA users [85], but although they were at a 'cool' house party, dancing was frequent, and a significant increase in body temperature was also found. In the present study, the Spearman correlations for the first dance club session demonstrated a positive association between saliva cortisol and hot and cold flushes $(\mathrm{r}=+0.88, \mathrm{p}=0.001)$, also with time spent drinking $(\mathrm{r}=+0.59, \mathrm{p}=0.044)$. This may indicate a common factor of thermal and fluid control, with HPA axis neuroendocrine activity. However the finding should be treated with caution, since it was not repeated at the second dance club session. In addition the sample size was very small, and a large number of correlations were being performed. These and other potential individual difference factors need to be investigated more fully in future studies. Cortisol is influenced by circadian or time-of-day factors, with a strong peak 1-2 $\mathrm{h}$ after waking, followed by comparatively low levels over the rest of the day [50]. In this study the circadian factor was well controlled, with times of testing very similar between the 2 drug conditions. Future studies could however investigate the complete 24-hour profiles of Ecstasy users. Finally, it may be noted that the current findings show that cortisol does not necessarily indicate negative psychological 'stress', since the marked rise in cortisol was accompanied by feelings of excitement, happiness and reduced anxiety (table 2). This is consistent with cortisol primarily being an energizing hormone [47].

The testosterone levels rose significantly following acute MDMA, whereas they were unchanged under MDMA abstinence (table 1; fig. 1). We believe that this is the first study to investigate testosterone changes under Ecstasy/MDMA, since a literature search revealed no previous empirical research involving either humans or laboratory animals. The acute increase in testosterone is consistent with Ecstasy's street name as the 'love drug' [5, 11,13 ] and 'love pill' attributed to the pharmacologically similar methylenedioxyamphetamine [51]. Sexual inter- 
course tends to be prolonged by the ring-substituted amphetamine derivatives MDMA and methylenedioxyamphetamine, with orgasm and ejaculation being delayed. Hence many recreational users state that ecstasy/MDMA is beneficial for sex, although others described it more as a sensual than a sexual drug $[13,14,52]$. Since MDMA increases cortisol (fig. 1), an acute rise in other corticotrophic hormones such as testosterone might be expected (fig. 2), given the close inter-relationship between the HPA and hypothalamic-pituitary-gonadal axes [50]. The present study showed a slight rebound for increased testosterone mid-week (fig. 1). Although this was statistically non-significant, it may relate to the significant midweek aggression noted in some ecstasy users $[17,18]$. Future studies should assess these hormonal and mood/ aggression changes in parallel. The current neuroendocrine findings were also analyzed with gender as an ANOVA factor, although they should be treated with caution given the small number of participants. Gender had minimal influence on the cortisol ANOVA results, which showed very similar patterns for males and females. With the testosterone data, there was a significant interaction between gender and time, although their different patterns of change over the 5 time points did not fit any clear pattern, so that as with the cortisol findings, males and females both showed marked increases in testosterone after MDMA. Future studies should however investigate the influence of gender in larger representative samples. It would also be illuminating to clarify the effects of MDMA on 'female' sex hormones such as progesterone.

\section{Thermal Aspects}

There was a slight (non-significant) increase in mean body temperature $2.5 \mathrm{~h}$ after MDMA of $0.15^{\circ} \mathrm{C}$, whereas in the equivalent abstinence condition the mean body temperature was significantly reduced by $0.19^{\circ} \mathrm{C}$ (table 1). These difference scores may partially reflect the slightly higher (although non-significant) baseline temperature value in the abstinence condition (fig. 2). The trend for higher body temperatures after MDMA was similar to previous findings. Some laboratory studies have found that acute MDMA leads to a significant increase in body temperature [37,38], whereas other laboratories have reported non-significant group differences, although with trends for higher temperatures following MDMA. For instance, Vollenweider et al. [40] reported that acute MDMA led to a: 'discrete increase of body temperature of about 0.2 to $0.5^{\circ} \mathrm{C}$, which however did not reach statistical significance'. Grob et al. [39] and Mas et al. [26] similarly mentioned non-significant trends to- wards an increase in body temperature. In one field study, Cole et al. [42] reported no temperature effects in a group of 'psychostimulant'-using dance clubbers, which included Ecstasy/MDMA polydrug users. Irvine et al. [41] found moderate increases in body temperature in those with the highest levels of plasma MDMA, several hours after a dance party. In other field studies, we have observed significant temperature increases in Ecstasy using dance clubbers [43] and Ecstasy/MDMA users at a house party [85]. Turning to the ambient temperature values, these rose significantly over time under both drug conditions (table 1), possibly due to the body heat generated within crowded dance clubs. This agrees with an earlier study of dance clubbers, where the ambient temperature increased significantly as the night progressed [42].

There was a highly significant rise in feeling hot when on Ecstasy/MDMA (table 1). This agrees with previous findings, where the majority of Ecstasy users report feeling hot on drug $[13,54]$. Hence in a recent Internet survey of more than 200 recreational Ecstasy users, $84 \%$ reported that they felt hot when on MDMA, and a small minority mentioned feeling very hot [32]. The present study also found a significant increase in the incidence of hot and cold flushes following MDMA, but no such trend was apparent when clubbing during MDMA abstinence (table 1). This therefore seems to be a specific effect of MDMA, rather than being a consequence of prolonged dancing in the club environment. It is consistent with the extensive animal literature on MDMA interfering with homeostatic thermal control mechanisms $[7,8,55]$. Finally, there does seem to be a degree of individual variation in the thermal experiences under MDMA. In the current study, 1 female participant reported feeling cold, was shivering, and wore scarves and extra clothing in attempt to feel warmer; yet her body temperature following MDMA remained unchanged from baseline.

\section{Mood State Changes}

There were significant mood gains under recreational MDMA, with significantly greater feelings of excitement, sociability and happiness compared to baseline (table 2). These positive mood changes were similar to previous findings $[13,16,18,56,57]$. There were no significant mood changes under MDMA abstinence, although several of the trends were in a positive direction - for instance with greater feelings of excitement (table 2). This may help to explain why happiness and excitement did not differ significantly between the on-MDMA and abstinence conditions, although there were significantly fewer feelings of depression and boredom at 1 and $2.5 \mathrm{~h}$ 
after MDMA respectively (table 3). This illustrates the importance of including an abstinence condition when evaluating the mood effects of MDMA. In a previous investigation of dance clubbers, we found positive mood profiles in both Ecstasy users and non-users, also noting that most people would expect to feel good on their Saturday night out irrespective of any drug-taking [18]. Hence part of the pleasant feelings attributed to MDMA may reflect the pro-social environment in dance clubs, along with general expectancy factors [56-58]. Mid-week mood decrements were apparent, with significantly lower feelings of excitement on day 4 compared to baseline, and significant tiredness 2 and 4 days after MDMA (tables 2 and 3). This agrees with previous findings of mood impairments and other psychobiological deficits midweek $[16-19,32,33,54,59,60]$.

\section{Problems Attributed to Ecstasy/MDMA}

Each participant described at least 1 psychobiological or health problem which they attributed to Ecstasy/ MDMA (table 4). The nature of these problems tended to alter with lifetime usage. Mood swings in the days afterwards were the main focus of the novice users, although mid-week mood fluctuation and depression were also noted by the more experienced consumers (table 4). Midweek recovery problems have been described in numerous studies, with feelings of depression, tiredness and anger, seen as a routine consequence of weekend Ecstasy use [16-19]. Mood fluctuation was the most frequent subjective complaint in an Internet study of 282 ecstasy users [60], as it was in the follow-up trial involving 209 further consumers [46]. Memory problems were not reported by any of the 5 novice users here, whereas they were noted by 6 of the 7 who had taken MDMA on 20 or more occasions (table 4). Memory and other cognitive deficits have been found on a wide variety of task measures [61-70], with the incidence and/or severity of these cognitive deficits being associated with lifetime Ecstasy usage [71-73]. Prospectively the cognitive-memory deficits also worsen with continued usage, whereas the cognitive decline is halted by quitting MDMA [74, 75]. Subjective complaints of memory problems are also associated with lifetime usage $[46,60]$. The other psychobiological problems noted here involved reduced food intake, as well as poorer appetite and sleep (table 4); again they are consistent with previous reports $[11,14,22,76,77]$. Another complaint was an exacerbation of pre-menstrual tension (table 4); this has been noted with other drugs such as nicotine [78, 79], and it should be further investigated with MDMA.

\section{Ethical Aspects}

Although MDMA is an illegal drug, its psychobiological effects in humans have been investigated in many previous empirical studies, and their ethical practices provided a guideline for the current analysis. Oral doses of MDMA have been given to human volunteers in many medical and laboratory studies, in order to measure its effects on cortisol [24-28], body temperature [37-39] and other psychobiological functions [review: 2]. Some of these studies have involved volunteers previously naïve to MDMA [40], although the majority administered MDMA to recreational Ecstasy users after they had agreed to undertake a brief period of abstinence [24-28, 37-39]. For our abstinence condition we similarly requested our experienced Ecstasy users to abstain for a brief period. Other empirical studies have tested recreational Ecstasy/MDMA in the real world environment of dance clubs and raves, comparing them to other illicit drug users and/or non-users as controls [1, 16-19, 41-43]. We therefore followed the ethical procedures which have become established practice within this field of applied research. For instance, the signed agreement form stated that MDMA was an illegal drug, that it had potentially damaging properties and that taking part in this study should not be seen as providing any support or encouragement for its use (see 'Methods'). The unpaid volunteers were interviewed and screened before acceptance into the study and informed that they were free to withdraw at any time without giving any reasons.

Some of the ethical and safety considerations were novel for this study, following the recommendations of the ethics committee. In particular, all the participants were known to the experimenter beforehand, since this was deemed preferable to obtaining volunteers via advertisements. The study was also fully discussed with each participant beforehand. Data collection was undertaken by 2 testers, so the ratio of testers-to-tested (in groups of 5 or 7) was always high. This provided mutual support for the data collectors and allowed the close monitoring of all participants, so facilitating intervention had any medical concerns arisen. Post-study feedback sessions were also provided. These had potential benefits to the volunteers in terms of increased practical knowledge. Indeed several of the participants were surprised at the cortisol findings in particular (fig. 1), since they graphically illustrated the power of MDMA as a psychoactive drug. This type of information could be useful for health education packages, since drug users often report that they seek scientific knowledge from 'real-world' studies such as this. 


\section{Future Research}

The present study illustrates the importance of conducing research in the dance club environment [16-19]. Future research could assess factors such as loud noise and overcrowding, since they may contribute to metabolic stress, both acute and chronic $[4,11,31,37,80,81]$. Other potentially important non-drug factors include social interactions, setting and expectancy $[4,13,55,56$, 58]. The effectiveness of protective strategies, such as the use of antioxidant drugs, limiting physical exertion, and frequent visits to the chill-out room could also be empirically assessed at dance club venues [32-34]. Future studies might also include more objective assessment measures, e.g. cognitive tests of memory and attention $[17,18]$. Although it is important not to overload the participants with assessment measures, since that might disrupt normal patterns of socialization and dancing. It would also be crucial to include a non-user control group, since they could indicate any differences in baseline neuroendocrine functioning, and psychobiological reactions to prolonged physical exertion and dance clubbing.

In conclusion, this field study has generated important new data on bioenergetic stress and recreational Ecstasy/ MDMA [11]. The energetic stress model for humans was based on an animal model, which described how drug and non-drug factors modulated the psychobiological consequences of MDMA [81]. MDMA is a powerful stimulant, which increases metabolic activity in the pre-synapses and heightens neurotransmitter release, but this acute metabolic stress in the serotonergic pre-synapse re- gion can lead to oxidative stress and cellular damage [6, 80,81 . This metabolic overstimulation can be further heightened by the concomitant use of other CNS stimulants such as amphetamine. Many non-drug factors can also influence the equation, including thermal stress, prolonged exercise, inadequate rest, overcrowding, loud noise and other stimulatory events $[1,4,11,82]$. The core of the model is that acute metabolic stress will lead to more acute functional deficits $[11,31]$ and to more cumulative psychobiological distress in the longer term $[32,81]$. The marked increase in cortisol in MDMA-using clubbers here (fig. 1) may therefore add an important new element to the metabolic stress model $[11,83]$. Testosterone is also acutely boosted by MDMA, and other neurohormonal changes may be important as well, through common or interactive HPA axis pathways. Cortisol in particular may provide a useful index of both energetic stress and of the integrity of metabolic energetic coping mechanisms. It may also contribute to chronic effects of repeated MDMA, as noted by Gerra et al. [29]. Hence future studies should measure cortisol, since this neurohormone may be an important co-factor for the overall psychobiology of MDMA.

\section{Acknowledgements}

We would like to thank Sarah Cook for her assistance with the data collection. The cortisol, testosterone and MDMA tests were funded by NIDA grant RO1 DA-14910-01 to Professors Lynn Singer, Andy Parrott, Derek Moore and John Turner.

\section{References}

1 Bedi G, Redman J: Recreational Ecstasy use: acute effects potentiated by ambient conditions? Neuropsychobiology 2006;53:113.

2 Dumont GJ, Verkes RJ: A review of acute effects of 3,4-methylenedioxymethamphetamine in healthy volunteers. J Psychopharmacol 2006;20:176-187.

-3 Parrott AC: Human research on MDMA (3,4-methylenedioxy-methamphetamine) neurotoxicity: cognitive and behavioural indices of change. Neuropsychobiology 2000; 42:17-24.

4 Parrott AC: MDMA (3,4-methylenedioxymethamphetamine) or Ecstasy: the neuropsychobiological implications of taking it at dances and raves. Neuropsychobiology 2004b;50:329-335.

5 Schifano F: Potential human neurotoxicity of MDMA ('Ecstasy'): subjective self-reports, evidence form an Italian drug addiction centre and clinical case studies. Neuropsychobiology 2000;42:25-33.
6 Zhou JF, Chen P, Zhou YH, Zhang L, Chen $\mathrm{HH}$ : 3,4-methylenedioxymethamphetamine abuse may cause oxidative stress and potential free radical damage. Free Radic Res 2003; 37:491-497.

7 Green AR, Mechan AO, Elliott JM, O’Shea E, Colado MI: The pharmacology and clinical pharmacology of 3,4-methylenedioxymethamphetamine (MDMA, 'Ecstasy'). Pharmacol Rev 2003;55:463-508.

8 Green A, O'Shea E, Colado I: A review of the mechanisms involved in the acute MDMA(Ecstasy)-induced hyperthermic response. Eur J Pharmacol 2004b;500:3-13.

-9 Morton J: Ecstasy: pharmacology and neurotoxicity. Curr Opin Pharmacol 2005;5:7986.

10 Carlezon WA Jr, Thome J, Olson VG, LaneLadd SB, Brodkin ES, Hiroi N, Duman RS, Neve RL, Nestler EJ: Regulation of cocaine reward by CREB. Science 1998;282:22722275 .
1 Parrott AC: MDMA in humans: factors which affect the neuropsychobiological profiles of recreational Ecstasy users, the integrative role of bioenergetic stress. J Psychopharmacol 2006;20:147-163.

12 Tancer ME, Johanson CE: The effects of fluoxetine on the subjective and physiological effects of 3,4-methylenedioxymethamphetamine (MDMA) in humans. Psychopharmacology 2007;189:565-573.

13 Cohen RS: The Love Drug. Marching to the Beat of Ecstasy. New York, Haworth Medical Press, 1998.

14 Parrott AC: Human psychopharmacology of Ecstasy (MDMA): a review of fifteen years of empirical research. Hum Psychopharmacol 2001;16:557-577.

15 Parrott AC: Is Ecstasy MDMA? A review of the proportion of Ecstasy tablets containing MDMA, dosage levels, and the changing perceptions of purity. Psychopharmacology 2004a;173:234-241. 
-16 Curran HV, Travill RA: Mood and cognitive effects of 3,4-methylenedioxymethamphetamine (MDMA, 'Ecstasy'): weekend 'high' followed by mid-week 'low'. Addiction 1997; 92:821-831.

-17 Curran VH, Rees H, Hoare T, Hoshi R, Bond A: Empathy and aggression: two faces of Ecstasy? A study of interpretative cognitive bias and mood change in Ecstasy users. Psychopharmacology 2004; 173:425-433.

-18 Parrott AC, Lasky J: Ecstasy (MDMA) effects upon mood and cognition; before, during, and after a Saturday night dance. Psychopharmacology 1998;139:261-268.

-19 Hoshi R, Pratt H, Mehta S, Bond AJ, Curran $\mathrm{HV}$ : An investigation into the sub-acute effects of Ecstasy on aggressive interpretative bias and aggressive mood - are there gender differences? J Psychopharmacol 2004;20: 291-301.

20 Jones K, Callen F, Blagrove M, Parrott AC: Self-rated sleep 7 days following recreational Ecstasy/MDMA use. J Psychopharmacol 2007;21:a28.

-21 McCann UD, Eligulashvili V, Ricaurte GA: 3,4-Methylenedioxymethamphetamine ('Ecstasy')-induced serotonin neurotoxicity: clinical studies. Neuropsychobiology 2000; 42:11-16.

22 Turner JJD, Nicolas L, Parrott AC: Reduced calorie intake in the week following weekend MDMA (Ecstasy) use. J Psychopharmacol 1998;12:a43.

-23 Harris DS, Baggott M, Mendelson JH, Mendelson JE, Jones RT: Subjective and hormonal effects of 3,4-methylenedioxymethamphetamine (MDMA) in humans. Psychopharmacology 2002;162:396-405.

-24 De la Torre R, Farre M, Roset PN, Lopez CH, Mas M, Ortuno J, Menoyo E, Pizarro N, Segura J, Cami J: Pharmacology of MDMA in humans. Ann NY Acad Sci 2000;914:225237.

-25 Farre M, de la Torre R, Mathuna BO, Roset PN, Peiro AM, Torrens M, Ortuno J, Pujadas M, Cami J: Repeated doses administration of MDMA in humans: pharmacological effects and pharmacokinetics. Psychopharmacology 2004; 173:364-375.

-26 Mas M, Farré M, De La Torre R, Roset PN, Ortuño J, Segura J, Camí J: Cardiovascular and neuroendocrine effects and pharmacokinetics of 3,4-methylenedioxymethamphetamine in humans. J Pharmacol Exp Ther 1999;290:136-145.

-27 Pacifici R, Zuccaro P, Farre M, Pichini S, Di Carlo S, Roset PN, Ortuno J, Segura J, de la Torre R: Immunomodulating properties of MDMA alone and in combination with alcohol: a pilot study. Life Sci 1999;65:309-316.

-28 Pacifici R, Zuccaro P, Farre M, Pichini S, Di Carlo S, Roset PN, Ortuno J, Pujadas M, Bacosi A, Menoyo E, Segura J, de la Torre R: Effects of repeated doses of MDMA ('Ecstasy') on cell-mediated immune response in humans. Life Sci 2001;69:2931-2941.
9 Gerra G, Bassignana S, Zaimovic A, Moi G, Bussandri M, Caccavari C, Brambilla F: Hypothalamic-pituitary-adrenal axis responses to stress in subjects with 3,4-methylenedioxy-methamphetamine ('Ecstasy') use history: correlation with dopamine receptor sensitivity. Psychiatry Res 2003;120:115124.

30 Parrott AC, Margetson J, Thome JC: Cigarettes, Ecstasy and the inverse of pleasure: nicotine abstinence effects in recreational Ecstasy/MDMA users. J Psychopharmacol 2005a;19:a27.

-31 Parrott AC: Recreational Ecstasy/MDMA, the serotonin syndrome, and serotonergic neurotoxicity. Pharmacol Biochem Behav 2002;71:837-844.

32 Parrott AC, Rodgers J, Buchanan T, Ling J, Heffernan T, Scholey AB: Dancing hot on Ecstasy: physical activity and thermal comfort ratings are associated with the memory and other psychobiological problems of recreational MDMA users. Hum Psychopharmacol 2006;21:285-298.

33 Topp L, Hando J, Dillon P, Roche A, Solowij $\mathrm{N}$ : Ecstasy use in Australia: patterns of use and associated harm. Drug Alcohol Depend 1999;55:105-115.

34 Tossman P, Boldt S, Tensil MD: The use of drugs within the techno party scene in European metropolitan cities. Eur Addict Res 2001;7:2-23.

35 Winstock AR, Griffiths P, Stewart D: Drugs and the dance music scene: a survey of current drug use patterns among a sample of dance music enthusiasts in the UK. Drug Alcohol Depend 2001;64:9-17.

-36 Suy K, Gijsenbergh F, Baute L: Emergency medical assistance during a mass gathering. Eur J Emerg Med 1999;6:249-254.

37 Freedman FR, Johanson C, Tancer ME: Thermoregulatory effects of 3,4-methylenedioxymethamphetamine (MDMA) in humans. Psychopharmacology 2005;183:248256.

38 Tancer ME, Johanson CE, Freedman RR: MDMA elevates core temperature in warm and cold conditions in man. Abstract Annu Conf Coll Probl Drug Depend, Florida, June 2003, p 169.

39 Grob CS, Poland RE, Chang L, Ernst T: Psychobiologic effects of 3,4-methylenedioxymethamphetamine in humans: methodological considerations and preliminary observations. Behav Brain Res 1996;73:103-107.

40 Vollenweider FX, Gamma A, Liechti M, Huber T: Psychological and cardiovascular effects and short-term sequelae of MDMA ('ecstasy') in MDMA naïve healthy volunteers. Neuropsychopharmacology 1998;19: 241-251.

41 Irvine RJ, Keane M, Felgate P, McCann UD, Callaghan PD, White JM: Plasma drug concentrations and physiological measures in 'dance party' participants. Neuropsychopharmacology 2006;31:424-430.
42 Cole JC, Sumnall HR, Smith GW, RostamiHodjegan A: Preliminary evidence of the cardiovascular effects of polysubstance misuse in night clubs. J Psychopharmacol 2005; 19:67-70.

43 Parrott AC, Young L: Increased body temperature in recreational Ecstasy/MDMA users out clubbing and dancing. J Psychopharmacol 2005;19:a26.

44 Parrott AC, Milani R, Parmar R, Turner JJD: Ecstasy polydrug users and other recreational drug users in Britain and Italy: psychiatric symptoms and psychobiological problems. Psychopharmacology 2001;159:77-82.

45 Milani RM, Parrott AC, Schifano F, Turner JJD: Patterns of cannabis use in Ecstasy polydrug users: moderate cannabis use may compensate for self-rate aggression and somatic symptoms. Hum Psychopharmacol 2005;20: $1-13$.

46 Rodgers J, Buchanan T, Pearson C, Parrott AC, Ling J, Heffernan TM, Scholey AB: Differential experiences of the psychobiological sequelae of Ecstasy use: quantitative and qualitative data from an Internet study. J Psychopharmacol 2006;20:437-446.

47 Rudolph DL, McAuley E: Self-efficacy and salivary cortisol responses to acute exercise in physically active and less active adults. J Sport Exerc Psychol 1995;17:206-213.

48 Davis H, Gass G, Bassett J: Serum cortisol response to incremental work in experienced and naïve subjects. Psychosom Med 1981;43: 127-132.

49 Kirwan J, Costill D, Flynn M, Mitchell J, Fink W, Neufer P, Houmard J: Physiological responses to successive days of intense training in competitive swimmers. Med Sci Sports Exerc 1988;20:255-259.

50 Lovallo WR: Stress and Health: Biological and Psychological Interactions. Thousand Oaks, Sage, 1997.

51 Jackson B, Reed A: Another abusable amphetamine. JAMA 1970;211:830.

52 Buffum J, Moser C: MDMA and human sexual function. J Psychoact Drugs 1986;18: 355-359.

53 Laloup M, Tilman G, Maes V, De Boeck G, Wallemacq P, Ramaekers J, Samyn N: Validation of an ELISA-based screening assay for the detection of amphetamine, MDMA and MDA in blood and oral fluid. Forensic Sci Int 2005; 153:29-37.

54 Davison D, Parrott AC: Ecstasy in recreational users: self-reported psychological and physiological effects. Hum Psychopharmacol 1997;12:91-97.

55 Green AR, Sanchez V, O'Shea E, Saadat KS, Elliott JM, Colado MI: Effect of ambient temperature and a prior neurotoxic dose of 3,4-methylenedioxy-methamphetamine (MDMA) on the hyperthermic response of rats to a single or repeated ('binge' ingestion) low dose of MDMA. Psychopharmacology 2004a;173:264-269. 
56 Greer G, Tolbert R: Subjective reports of the effects of MDMA in a clinical setting. J Psychoactive Drugs 1986;18:319-327.

57 Parrott AC: The psychotherapeutic potential of MDMA (3,4-methylenedioxymethamphetamine): an evidence-based review. Psychopharmacology 2007;191:181-193.

-58 Curran V: Is MDMA ('Ecstasy') neurotoxic in humans? An overview of evidence and of methodological problems in research. Neuropsychobiology 2000;42:34-41.

-59 Verheyden SL, Hadfield J, Calin T, Curran HV: Sub-acute effects of MDMA (+/-3,4methylenedioxymethamphetamine, 'Ecstasy') on mood: evidence of gender differences. Psychopharmacology 2002;161:23-31.

-60 Parrott AC, Buchanan T, Scholey AB, Heffernan TM, Ling J, Rodgers J: Ecstasy-attributed problems reported by novice, moderate and heavy recreational users. Hum Psychopharmacol 2002;17:309-312.

61 Rendell PG, Gray TJ, Henry JD, Tolan A: Prospective memory impairment in 'Ecstasy' (MDMA) users. Psychopharmacology 2007;194:497-504

62 Bolla KI, McCann UD, Ricaurte GA: Memory impairment in abstinent MDMA ('Ecstasy') users. Neurology 1998;51:1532-1537.

63 Fox HC, McLean A, Turner JJD, Parrott AC, Rogers R, Sahakian BJ: Neuropsychological evidence of a relatively selective profile of temporal dysfunction in drug-free MDMA ('Ecstasy') polydrug users. Psychopharmacology 2002;162:203-214.

-64 Krystal JH, Price LH, Opsahl C, Ricaurte GA, Heninger GR: Chronic 3,4-methylenedioxymethamphetamine (MDMA) use: effects on mood and neuropsychological function? Am J Drug Alcohol Abuse 1992;18: 331-341.

65 Morgan MJ: Memory deficits associated with recreational use of 'Ecstasy' (MDMA). Psychopharmacology 1999;141:30-36.
66 Reay JL, Hamilton C, Kennedy DO, Scholey $\mathrm{AB}$ : MDMA polydrug users show processspecific central executive impairments coupled with impaired social and emotional judgement processes. J Psychopharmacol 2006;20:385-388.

67 Rodgers J: Cognitive performance amongst recreational users of 'Ecstasy'. Psychopharmacology 2000;151:19-24.

68 Gouzoulis-Mayfrank E, Daumann J, Tuchtenhagen F, Pelz S, Becker S, Kunert HJ, Fimm B, Sass H: Impaired cognitive performance in drug-free users of recreational Ecstasy (MDMA). J Neurol Neurosurg Psychiatry 2000;68:719-725.

69 Parrott AC, Lees A, Garnham NJ, Jones M, Wesnes K: Cognitive performance in recreational users of MDMA or 'Ecstasy': evidence for memory deficits. J Psychopharmacol 1998;12:79-83.

70 Wareing M, Fisk JE, Murphy PN: Working memory deficits in current and previous users of MDMA ('Ecstasy'). Br J Psychol 2000; 91:181-188.

71 Halpern JH, Pope HG, Sherwood AR, Barry S, Hudson JI, Yurgelun-Todd D: Residual effects of illicit 3,4-methylenedioxymethamphetamine in individuals with minimal exposure to other drugs. Drug Alcohol Depend 2004;75:135-147.

72 Fisk JE, Montgomery C, Wareing M, Murphy PN: Reasoning deficits in Ecstasy (MDMA) polydrug users. Psychopharmacology 2005;181:550-559.

73 Fox H, Parrott AC, Turner JJD: Ecstasy/ MDMA-related cognitive deficits: a function of dosage rather than awareness of problems. J Psychopharmacol 2001;15:273-281.

74 Zakzanis KK, Young DA: Memory impairment in abstinent MDMA ('Ecstasy') users: a longitudinal investigation. Neurology 2001;56:966-969.

75 Zakzanis KK, Campbell Z: Memory impairment in now abstinent MDMA users and continued users: a longitudinal follow-up. Neurology 2006;66:740-741.
6 Schifano F, Magni G: MDMA ('Ecstasy') abuse: psychopathological features and craving for chocolate: a case series. Biol Psychiatry 1994;36:763-767.

77 Soar K, Parrott AC, Turner JJD: Persistent neuropsychological problems after seven years of abstinence from recreational Ecstasy (MDMA): a case study. Psychol Rep 2004; 95:192-196.

78 Craig D, Parrott AC, Coomber J: Smoking cessation in women: effects of the menstrual cycle. Int J Addict 1992;27:697-706.

79 Mishra GD, Dobson AJ, Schofield MJ: Cigarette smoking, menstrual symptoms and miscarriage among young women. Aust NZ J Public Health 2000;24:413-420.

80 Darvesh AS, Gudelsky GA: Evidence for a role of energy dysregulation in the MDMAinduced depletion of brain 5-HT. Brain Res 2005;21:168-175.

81 Huether G, Zhou D, Rüther E: Causes and consequences of the loss of serotonergic presynapses elicited by the consumption of 3,4-methylenedioxymethamphetamine (MDMA, 'Ecstasy') and its congeners. J Neural Transm 1997;104:771-794.

82 Parrott AC: Recreational Ecstasy use: acute effects potentiated by ambient conditions (author's reply). Neuropsychobiology 2006; 53:113.

83 Yau JL, Noble J, Seckl JR: Site-specific regulation of corticosteroid and serotonin receptor subtype gene expression in the rat hippocampus following 3,4-methylenedioxymethamphetamine: role of corticosterone and serotonin. Neuroscience 1997;78: 111-121.

84 Bergomi M, Rovesti S, Vivoli G: Biological response to noise and other physical stressors in places of entertainment. Public Health Rev 1991;19:263-275.

85 Parrott AC, Adnum L, Evans A, Kissling C, Thome J: Heavy Ecstasy/MDMA use and cool house parties: substantial cortisol release and increased body temperature. J Psychopharmacol 2007;21:a35. 